\title{
La institucionalidad deportiva argentina para jóvenes y mujeres: vínculos con los
} derechos humanos y la inclusión social

Yussef Becher ${ }^{1}$

CONICET/ Universidad Nacional de San Luis

\section{Ensayo}

Material original autorizado para su primera publicación en el Journal de Ciencias Sociales, Revista Académica de la Facultad de Ciencias Sociales de la Universidad de Palermo

Recepción: 01-08-2017

\section{Aceptación: 09-03-2018}

Resumen: El artículo propone una particular mirada sobre la institucionalidad deportiva argentina de juventudes y mujeres en donde priman los conceptos de derechos humanos e inclusión social. Tales significantes se han vuelto recurrentes por parte de discursos políticos y mediáticos, de allí la necesidad de las ciencias sociales de definir los elementos teóricos que los componen, superando todo tipo de banalidades. En ese sentido se orienta nuestra propuesta teórico-metodológica respecto de este texto.

En cuanto a los aspectos metodológicos, acudimos a una estrategia de corte cualitativa y teórica. Dicha estrategia consiste en definir los elementos teóricos que conforman un concepto y analizar los documentos oficiales sobre el tema u otra unidad de análisis que integre la investigación. A partir de ello, se contrastarán tales aspectos teóricos con los datos emergentes de los documentos. Por ello es una estrategia en parte teórica y en otra muy importante inductiva.

Los resultados de la exploración muestran que la institucionalidad deportiva argentina para jóvenes y mujeres -en su tratamiento por parte de los documentos oficiales- oscila en torno a los conceptos de derechos y meritocracia. Por una parte, encontramos programas sociales que, en términos de derechos, significan un importante avance en la materia, pero, al mismo tiempo, contienen disposiciones que restringen su posibilidad de mayor acceso. Por otra parte, encontramos disposiciones sobre dicha institucionalidad en donde prima una lógica de funcionamiento meritocrática en la que se selecciona de entre posibles postulantes 0 instituciones a las que desde aquella concepción son consideradas las mejores.

\footnotetext{
${ }^{1}$ Abogado. Magister en Sociedad e Instituciones. Doctorando en Ciencias Sociales (FLACSO Argentina). Becario doctoral del Consejo Nacional de Investigaciones Científicas y Técnicas (CONICET). Docente investigador de la Facultad de Ciencias Económicas, Jurídicas y Sociales (FCEJS). Universidad Nacional de San Luis (UNSL). Correo electrónico: yussefbe@hotmail.com
} 
Palabras clave: institucionalidad deportiva; jóvenes; mujeres; derechos humanos.

\title{
The Argentinian sport institutionality for young people and women: links with human rights and social inclusion
}

\begin{abstract}
The article proposes a particular view at the Argentine sports institutions of youths and women, where the concepts of human rights and social inclusion prevail. Such signifiers have become recurrent on the part of political and media discourses and from there the need of the social sciences to define the theoretical elements that compose them surpassing all type of banalities. In this sense, our theoretical-methodological proposal is oriented towards this text.
\end{abstract}

As for the methodological aspects, we turn to a qualitative and theoretical strategy. This strategy consists of defining the theoretical elements that constitute a concept and analyzing the official documents on the subject or another unit of analysis that integrates the research. From it the possibility of contrasting such theoretical aspects with the data emerging from the documents. For this reason, it is a strategy in part theoretical and in another very inductive part.

The results of the exploration show that Argentine sports institutions for young people and women -in their treatment by the official documents- oscillate around the concepts of rights and meritocracy. On one side, we find social programs that in terms of rights signify an important advance in the matter but, at the same time, contain provisions that restrict their possibility of greater access. On the other side, we find provisions on this institutionality where a meritocratic logic of operation in which is selected from among potential applicants or institutions that from that conception are considered the best.

Keywords: sports institutionality; young; women; human rights.

\section{Introducción}

Es recurrente escuchar tanto en los medios de comunicación argentinos como en los políticos, el uso de significantes tales como derechos humanos e inclusión social. Aunque esto no solo sucede en nuestro país, pues -tal como comenta Ruth Levitas (2006)- los conceptos de exclusión e inclusión social también se encuentran arraigados en la política y el gobierno británico y europeo, principalmente, a partir de la influencia de los organismos internacionales. De modo que a las ciencias sociales les corresponde la función que siempre ha interpelado a sus actores: la de definir con rigurosidad científica los elementos teóricos 
que conforman a un concepto o a un conjunto de categorías teóricas. En ese sentido, el texto propone -respecto de una temática particular- una propuesta teórica y metodológica acorde.

En los últimos quince años de la contemporaneidad argentina, esos significantes (derechos e inclusión) han estado presentes principalmente por medio de políticas sociales $y$, entre ellas, las acciones deportivas. Tales políticas pueden ser indagadas desde una doble perspectiva: la de su diseño o la de su implementación. En nuestro texto proponemos bucear en esa segunda instancia, que comprende al conjunto de instituciones involucradas en la ejecución del programa social, y respecto de la propuesta deportiva para jóvenes y mujeres.

Tanto la juventud, como etapa cronológica social, como la identidad sexual pueden formar parte de una misma subjetividad. Por ello, en principio no es correcto tratarlas por separado, pero son los mismos documentos oficiales que analizamos los que lo hacen de ese modo y las mismas políticas sociales las que han dividido la cuestión. Pues podemos distinguir claramente -y lo hacemos en una de las secciones del artículo, citando estadísticas e informes de organismos internacionales- entre políticas sociales propiamente juveniles y otras que tienen por objetivo la reducción de brechas de género. Ambas tienen por fin igualar condiciones, materiales y simbólicas, ya sean las juveniles o las de género.

En cuanto a los aspectos metodológicos, el análisis de la institucionalidad se realiza por medio de una estrategia cualitativa y teórica. Dicha estrategia tiene como principal objetivo definir los elementos teóricos que conforman a un concepto (en nuestro caso derechos humanos e inclusión social) y explorar en documentos oficiales su tratamiento y la posible emergencia de otros conceptos vinculados. Tales documentos son definidos por Maurice Duverger (1981) como aquellos que provienen de los poderes constituidos de un Estado. Si bien hemos revisado diversos documentos oficiales, la mayoría del análisis se centra en una normativa reciente (la Ley № 27201 del año 2015) que constituye un intento por articular diferentes instituciones deportivas. Al mismo tiempo, crea e introduce modificaciones en programas sociales con trayectorias ya consolidadas (como la Asignación Universal por Hijo para Protección Social) y nuevos como el Programa Nacional de Empoderamiento de la Mujer en el Deporte.

Los resultados de la exploración realizada sobre tales documentos muestran que la institucionalidad deportiva para jóvenes y mujeres incorpora importantes aspectos ligados a la inclusión social, con especial referencia a los derechos humanos, pues establece una adecuada coordinación de tareas entre los diferentes niveles de gobierno y medidas que pueden contribuir a la igualación de las condiciones de vida en términos de juventudes y género. Sin dudas, persiste una modalidad meritocrática de acceso a determinados recursos en el área deportiva y, en el marco de dicha institucionalidad, existen intentos también por 
morigerar esa modalidad. No obstante, aun no se ha explicitado en los documentos oficiales el detalle de las acciones propuestas para ello.

\section{Derechos humanos e inclusión social}

Tales derechos se vinculan con la inclusión social por cuanto el derecho a la igualdad y no discriminación impone la obligación de cumplir de parte del Estado con la inclusión mediante diversas estrategias- de todos los sectores de la sociedad y especialmente de aquellos que padecen situaciones de desigualdad. Asimismo, todo derecho humano se compone de dos dimensiones: una relativa a su vigencia y la otra a su efectividad. La primera de ellas refiere al aspecto positivo y su regulación por medio de normas jurídicas y la segunda a la dimensión material o sustantiva. La última es la que incorpora el aspecto relativo al contexto social, por cuanto supone la aplicación de la norma en la realidad social. De allí que formalmente el derecho a la igualdad y no discriminación se encuentra reconocido en los diferentes tratados internacionales que integran tanto el sistema universal como el interamericano de derechos humanos. Si bien las perspectivas positivistas o formalistas han permeado con mayor predominancia durante muchos años el campo de estudios en materia de derechos, desde hace ya también varios años se ha propuesto incorporar en su estudio sus aspectos materiales o sustanciales. En materia de inclusión, ello supone considerar las condiciones materiales y simbólicas reales de quienes deben ser incluidos. A este aspecto lo hemos denominado -tomando como influencia teórica el planteo de Delor y Hubert (2000)dimensión subjetiva, por cuanto procuramos reparar en los efectos de los procesos inclusivos sobre los sujetos a incluir (Becher, 2018). Tanto la condición de empleo como la de sociabilidad -tal como plantean las investigaciones sociales francesas sobre el tema, y entre ellas principalmente la de Castel (2004)- son dos aspectos que definen el grado de integración social de los ciudadanos. El aspecto referido a la sociabilidad es clave, pues los efectos de la inclusión sobre las subjetividades son reparar o construir nuevas redes de sociabilidad respecto de quienes han estado sometidos a algunas de las diferentes modalidades de la exclusión. Esto mismo es reflejado en los textos de Peter Towsend (1979) y en el más reciente de Burchardt et. al. (2002). El primero de ellos afirma que una comprensión adecuada de la pobreza no debe restringirse al análisis de necesidades elementales no satisfechas, pues debe incorporar la incapacidad de las personas para participar en la vida habitual de la sociedad. Por su parte Burchardt et. al. (2002) afirma: 
un individuo está excluido socialmente si no forma parte de actividades clave de la sociedad en la cual vive... el individuo no participa de las mismas por razones más allá de su control (en referencia a la exclusión) pero le gustaría participar (pp. 30-32). ${ }^{2}$

Tanto en un fragmento como en otro la dimensión referida a la sociabilidad de los sujetos es un aspecto clave, en el caso del primero para la concepción de la pobreza y en el del segundo para la de exclusión. De modo que si la pobreza o la exclusión dañan la cohesión social o los vínculos sociales establecidos, por consiguiente a la inclusión le corresponde contrarrestar tales efectos.

Al inicio del párrafo precedente mencionábamos que el Estado acude a diferentes estrategias para promover procesos inclusivos, y en nuestro país, como en gran parte de la región latinoamericana, en los primeros quince años del siglo XXI tales estrategias se han objetivado por medio de políticas sociales. Dichas políticas pueden ser analizadas desde alguna de sus ya tradicionales esferas de indagación: la de su diseño o la de su implementación. La segunda de ellas es la que comprende al conjunto de instituciones involucradas en su ejecución y es comúnmente denominada institucionalidad social (Oszlak y O'Donnell, 1995). En este texto proponemos bucear en esa dimensión de la política social (a partir del análisis de los documentos oficiales) desde una mirada vinculada a los derechos humanos y a la inclusión social.

Vale señalar que observar tal dimensión de la política social respecto del deporte constituye un desafío, pues la institucionalidad social en esa materia ha sido objeto de diversas modificaciones en estos últimos quince años, sin embargo, hemos encontrados dos principales incentivos: por una parte, los propios documentos oficiales en donde se señala que su diseño ha sido realizado desde el enfoque de derechos e inclusión y, por otra, una normativa en donde se intentan articular y organizar diferentes instituciones involucradas en dicha área, principalmente respecto de las juventudes y las mujeres.

\section{Políticas de juventudes y de reducción de brechas de género en contexto}

Desde inicios del siglo XXI en nuestro país la inversión en políticas sociales destinadas a las juventudes y la reducción de brechas de género se incrementó considerablemente. En el año 2014, el Instituto Nacional de Estadísticas y Censos (INDEC) realizó por primera vez un relevamiento al que denominó Encuesta Nacional de Jóvenes cuyos resultados fueron publicados durante el año 2015. Dicho relevamiento comprendió entre su población a jóvenes residentes en centros urbanos de todo el país de 2000 o más habitantes, quienes fueron seleccionados de acuerdo con un criterio etario de 15 a 29 años.

\footnotetext{
2 Traducción propia; el agregado entre paréntesis nos corresponde.
} 
Los resultados muestran importantes niveles de finalización e inserción respecto de la educación y el empleo. Del total de la muestra, un $40 \%$ de los jóvenes de 20 a 29 años de edad han finalizado la educación media en el tiempo previsto, mientras que un $28 \%$ del total de jóvenes de 15 a 29 años de edad asisten actualmente al secundario. Tales datos permiten aseverar que en Argentina existe un elevado índice de alfabetización juvenil y de trayectorias educativas de nivel secundario completas. En lo que respecta al empleo, el $45.6 \%$ de los jóvenes afirmó trabajar y, de entre ellos, un 34\% se dedica a tareas de cuidado -ya sea respecto de su entorno familiar o de otros- con una significativa diferencia porcentual entre varones y mujeres. Aproximadamente 3 de cada 10 jóvenes que se dedican a tareas de cuidado dejan de trabajar o estudiar, y el porcentaje es de $42.1 \%$ para las mujeres y de $12.9 \%$ para los varones. Ello muestra nuevamente la persistente existencia de brechas de género (en tanto acceso desigualitario a los recursos) y la reproducción de estereotipos respecto del empleo en las mujeres.

La Organización Iberoamericana de la Juventud (OIJ) y la Comisión Económica para América Latina y el Caribe de la Organización de Naciones Unidas (CEPAL-ONU) también realizaron un relevamiento en el año 2015. Ambos organismos señalan que en Argentina habitan un total de 10.050.434 jóvenes varones y mujeres de entre 15 y 29 años de edad. Ello representa un $24.7 \%$ sobre el total poblacional calculado por tales organismos, y de ese total el $2.86 \%$ son considerados pobres y el $1.75 \%$ son indigentes. En cuanto a la educación y el empleo, el $83.3 \%$ ha concluido su educación básica y el 14\% se encuentra desempleado.

Los mismos organismos señalan que la inversión social en políticas de juventud por parte del gobierno argentino era de un 3.3\% sobre el Producto Bruto Interno (PBI), lo que la colocaba en una diferencia porcentual de $25 \%$ respecto del promedio de los otros países de la región. Dicho porcentaje de inversión se distribuía en políticas sociales mayoritariamente educativas y eran seguidas por las de vivienda, salud y asistencia social. Asimismo, el informe destaca la importante inversión en programas de transferencias condicionadas dirigidos a las juventudes, tales como la Asignación Universal por Hijo o el Programa de Respaldo a Estudiantes Argentinos.

En el año 2013, el Consejo Latinoamericano de Ciencias Sociales (CLACSO), en conjunto con la Organización de las Naciones Unidas para la Educación, la Ciencia y la Cultura (UNESCO), realizaron un relevamiento sobre el tipo de políticas púbicas de juventud en las que invertía Argentina. Del total de programas sociales -88 en números absolutos-, el $30.7 \%$ estaban destinados a la población joven, mientras el $69.3 \%$ no las comprendía como destinatarias directas (pues estaban dirigidos a una población más amplia), pero sí por sus franjas etarias. Del total, el 25\% se concentraba en el Ministerio de Desarrollo Social; el 13.6\% en el Ministerio de Industria; el 11.4\% en el Ministerio de Educación; el $8 \%$ en la Jefatura de 
Gabinete de Ministros; y siguen porcentajes menores en otras carteras ministeriales. De entre el conjunto de políticas de juventudes, el mayor número se ubicaba en las que se denominan de participación social y ciudadana, y a continuación, con poca diferencia, se ubicaban las de promoción del desarrollo económico y de acceso a derechos y actividades culturales. Por último, y con porcentajes menores, se encontraban las políticas de acceso y terminalidad educativa y las de inclusión social. Tales denominaciones corresponden a las contenidas en los documentos oficiales de las respectivas programaciones, pues muchas de ellas podrían ser comprendidas por la categoría inclusión social.

Vale mencionar que en el año 2015 el Instituto de Investigaciones Gino Germani de la Universidad de Buenos Aires realizó un relevamiento de similares características y fue publicado en Vázquez (2015). En dicho estudio el número de programas sociales asciende a 150 por considerar aquellos en los cuales los jóvenes no son sus destinatarios directos (pues la población de la política está definida en términos más amplios), pero por las edades comprendidas obtienen el acceso a los derechos establecidos en los programas. Si bien en este estudio la tipología construida es más detallada y exhaustiva, la mayor cantidad de programaciones se concentraba en las mismas categorías del estudio anterior.

En ambos estudios se menciona que, si se consideraran la cantidad de destinatarios, el impacto o el presupuesto destinado a cada una de ellas, la distribución sería distinta, pues el primer lugar correspondería sin dudas a las de inclusión social (que comprenden las transferencias condicionadas) y las de terminalidad educativa.

En materia de brechas de género es la CEPAL el organismo que con mayor periodicidad realiza relevamientos en el marco del sistema universal de protección de derechos humanos. Dicho organismo creó en el año 2007, en la décima Conferencia Regional sobre la Mujer de América Latina y el Caribe, el Observatorio de Igualdad de Género. Entre sus tareas se encuentra la de ofrecer a la comunidad datos estadísticos ligados con tales brechas y lo hace en función de cuatro variables: la autonomía económica; la autonomía en la toma de decisiones; la autonomía física; y la interrelación de las autonomías. El primer indicador muestra que en nuestro país el $58.5 \%$ de las mujeres no cuenta con ingresos propios y de su tiempo dedica semanalmente un $43 \%$ al trabajo no remunerado. El segundo indicador muestra que en Argentina los cargos ministeriales corresponden en un 19\% a las mujeres. Asimismo, el porcentaje de mujeres en la Corte Suprema de Justicia de la Nación es de $28.6 \%$ y en el Poder Legislativo de $35.8 \%$. Los dos primeros porcentajes colocan a nuestro país en una posición media respecto de los otros de la región, pero el último entre los primeros. Por su parte, el indicador autonomía física muestra que Argentina tiene una de las tasas más elevadas de feminicidios y de muerte de mujeres ocasionadas por sus parejas 
o ex parejas íntimas. El índice de maternidad adolescente la ubica en la media respecto de los otros países y el de mortalidad materna entre los de menor porcentaje.

El Foro Económico Mundial estima anualmente el Índice Global de la Brecha de Género y, respecto del año 2016, Argentina se ubica en el puesto número 33 con un porcentaje del $73.52 \%$. Ello muestra que en nuestro país (de acuerdo con los indicadores considerados que son similares a los de la CEPAL) la brecha no es amplia entre varones y mujeres respecto de los otros países analizados en los cuales se encuentran representados los cinco continentes. Asimismo, nuestro país muestra una reducción sostenida de las desigualdades de género desde el año 2006 (donde inicia el registro del Foro) y los valores más bajos corresponden a los años 2008 y 2009. En ese último año se sancionó la Ley $N^{\circ}$ 26485 de Protección Integral de la Violencia contra las Mujeres.

Si bien Argentina no cuenta con un plan de igualdad de género implementado a nivel nacional -modalidad que se ha extendido en otros países de la región-, sí cuenta con políticas públicas sectoriales que tienen aquel fin. En lo que respecta al presupuesto destinado a programaciones de igualdad de género, entre los años 2015 y 2016 el monto designado al Consejo Nacional de Mujeres (de acuerdo con la ley de presupuesto) se ha incrementado en un $132 \%$. En lo relativo al marco normativo institucional, nuestro país cuenta desde el año 1991 con una ley de cupo femenino para cargos legislativos nacionales y provinciales, y recientemente con leyes sobre violencia integral, trata de personas, acoso sexual y una reforma al Código Penal en la que se incorporó el delito de femicidio (CEPAL, 2017).

El Comité para la Eliminación de la Discriminación contra la Mujer (que controla en cada Estado Parte el cumplimiento de los derechos contemplados en el tratado) en su último informe del año 2010 destacó la importante inversión argentina en el área de la protección y la seguridad social, y en ello su impacto positivo en la disminución de tasas de pobreza de mujeres y niñas como así también la prioridad asignada en tales ámbitos a las mismas.

En lo relativo a la participación de las mujeres en la vida pública, el Comité señaló la importancia de que por primera vez una mujer haya sido elegida presidenta por medio del voto popular (en referencia a la ex mandataria Cristina Fernández) y la significativa presencia femenina en cargos ministeriales y de la Corte Suprema de Justicia.

Por otra parte, también mencionó la importancia de que el Estado haya sancionado la Ley Integral de Violencia contra las Mujeres. Dicha ley establece medidas preventivas y crea el Observatorio de la Violencia. Al mismo tiempo, y en el ámbito de la Corte Suprema de Justicia de la Nación, se creó la Oficia de Violencia Doméstica.

En cuanto a los ámbitos en los que el Estado argentino debe implementar medidas para reducir desigualdades de género - de acuerdo con lo señalado por el Comité-, se 
incorporan aspectos relativos al acceso a la información, la mejora de la institucionalidad dirigida a las mujeres, la incorporación de la perspectiva de género en programas sociales, y otros relativos a la educación y el empleo. Con respecto al acceso a la información, se destaca la importancia de que los diferentes organismos públicos y sus agentes estatales conozcan el alcance de las leyes sobre género y las medidas que plantean para hacerlas efectivas. En lo relativo a la institucionalidad, recomienda que el Estado incremente la inversión en infraestructura para el Consejo Nacional de Mujeres -lo cual puede advertirse en el informe de la CEPAL del año 2017- y demás organismos vinculados con los derechos de las mujeres. Los programas sociales constituyen otro ámbito en el que se recomienda la incorporación de la perspectiva de género, por cuanto muchos de ellos establecen condicionalidades donde las mujeres son las encargadas de cumplirlas y, de ese modo, contribuyen a una desigual distribución de las tareas de cuidado respecto de los varones. En materia de educación y de empleo, las principales recomendaciones giran en torno a la necesidad de interpelar a las mujeres (desde la educación) a instancias de socialización que superen los estereotipos de género, de allí la posibilidad de insertarse en empleos distintos de aquellos que comúnmente les son asignados.

\section{Algunas precisiones metodológicas: una estrategia de análisis teórico y cualitativo}

Tal como se señaló anteriormente, las estrategias de parte del Estado para promover procesos inclusivos se han objetivado principalmente en políticas sociales y una de sus posibles dimensiones de análisis es la de la institucionalidad. En tal sentido, en el texto nos centramos en esa dimensión referida al deporte y hemos seleccionado una estrategia de análisis teórica y cualitativa.

En ciencias sociales, cuando se ha acudido a tales estrategias, por lo general se han revisado los aportes teóricos de autores clásicos en dicha área. Nuestra propuesta, en cambio, se vincula con la posibilidad de definir los elementos teóricos que conforman un concepto (en nuestro caso, derechos humanos e inclusión social) y de allí explorar el modo en que se ha realizado su tratamiento por parte de documentos oficiales. Ello muestra que no es una propuesta distinta de las propias de las investigaciones cualitativas, aunque la interacción de la construcción del marco teórico y el trabajo empírico se produce entre ese marco de referencia y el contenido de los documentos. Por ello, es en parte teórica y en otra muy importante, inductiva.

$\mathrm{Si}$ bien dicha estrategia comparte algunas características con los análisis documentales, supera los fines descriptivos (no por ello menos analíticos) que caracterizan a aquellos e intenta profundizar en el contenido de los datos expresados en los documentos. Para ello es necesario construir minuciosamente un contexto conceptual en donde las 
principales estrategias son una búsqueda exhaustiva de bibliografía sobre el tema y la posibilidad de incorporar los conocimientos propios del investigador sobre la temática (Maxwell, 1996). Vale señalar que un aspecto relevante en torno a la elaboración de dicho contexto es la construcción de un argumento que lo sostenga. De allí que sugerimos particularmente considerar el contexto sociopolítico en que fueron implementados los documentos que se analizan. Tal como plantea Tonon (2015), toda investigación cualitativa debe considerarlo y propone el término indexicality: “... revela el significado contextual y muestra la dependencia de los actos concretos con el contexto situacional en que se producen; por lo tanto, solo pueden explicarse si se ha entendido el contexto" (p. 7$)^{3}$.

Con el fin de profundizar dicha estrategia teórica y cualitativa, una posible herramienta de estudio de los datos es la que propone el análisis temático. Ese tipo de técnicas son propias de las investigaciones cualitativas, ya sean teóricas o empíricas. En nuestro caso hemos decidido guiarnos por los pasos propuestos para ese tipo de análisis por las investigadoras en metodología cualitativa Braun y Clark (2006). En el primero de ellos se realizan comentarios generales sobre los documentos seleccionados. Si bien hemos revisado diferentes documentos sobre institucionalidad deportiva en Argentina, optamos por la Ley $\mathrm{N}^{\circ}$ 27201 -que crea el Ente Nacional de Desarrollo Deportivo- por motivos que anteriormente hemos comentado y profundizaremos en el próximo apartado.

En el segundo paso del análisis temático se definen los primeros códigos que surgen de la relación entre la teoría y los datos emergentes de los documentos. Del mismo modo, en los siguientes pasos es donde se definen con mayor detalle tales códigos y se establecen las relaciones jerárquicas entre ellos. De allí que hemos seleccionado principalmente dos temas que comprenden diferentes sub temas: institucionalidad e inclusión. Ya hemos explicitado anteriormente el modo en que se vinculan teóricamente tales conceptos con el de derechos humanos y ahora lo haremos a partir del análisis de los documentos. En la última etapa de dicho análisis se redacta el informe final de acuerdo con los temas y sub temas definidos anteriormente.

\section{Análisis de la normativa: entre los derechos y la meritocracia}

Que una normativa y la institucionalidad a la que da origen se vinculen con las juventudes y las mujeres no es baladí, pues tal como hemos señalado anteriormente ambos sectores poblacionales han formado parte en las últimas décadas de sendas intervenciones estatales. Ello, por una parte, muestra la intención del gobierno de reparar a sectores sociales históricamente postergados (más allá de los beneficios que reportan para ese gobierno) y,

\footnotetext{
3 Traducción propia.
} 
por otra, una orientación ideológica en donde la inclusión por medio del acceso a derechos es uno de los objetivos del proyecto político. Aunque -tal como destaca Andrenacci (2010)hacia fines de la primera década del siglo XXI la mayoría de los países de la región (incluso los de centro derecha) mostraron cierta sensibilidad hacia los sectores populares y principalmente los más postergados durante la década de 1990.

En dicho contexto se enmarca la Ley $N^{\circ} 27201$ que crea el Ente Nacional de Desarrollo Deportivo. Seleccionamos esa normativa por cuanto se trata de una propuesta reciente del Poder Ejecutivo (aprobada por el Congreso) correspondiente al año 2015, y un buen intento por articular diferentes instituciones y programas sociales deportivos dirigidos a jóvenes y mujeres. Entre ellos quedan comprendidos la Asignación Universal por Hijo para Protección Social (regulada por medio del Decreto $N^{\circ} 1602 / 09$ e incorporado a la Ley $N^{\circ}$ 24714), el Programa Nacional de Empoderamiento de la Mujer en el Deporte (creado por Ley $N^{\circ} 27201$ ), el Programa Social y Deportivo Juegos Nacionales Evita (regulado por Ley $\mathrm{N}^{\circ}$ 26462), el Programa de Becas Sociales Deportivas, y un conjunto de disposiciones generales dirigidas a regular diferentes aspectos de la institucionalidad deportiva argentina.

A partir de lo enunciado por los documentos oficiales, la inclusión social y los espacios de socialización propuestos aparecen asociados a los derechos humanos y particularmente a una de sus etapas: la de goce efectivo o de acceso a los mismos. En uno de los documentos se afirma:

El ente tiene plena capacidad jurídica para administrar los recursos asignados en la presente ley, afectándolos exclusivamente a... e) promocionar los valores del deporte y la educación física, implementando planes y programas que permitan el acceso a la práctica de los deportes, contemplando el ámbito rural y urbano, en especial de los niños, niñas y adolescentes, considerando la animación sociocultural como auténtico medio de inclusión y estabilidad social (art. 10 de la Ley $N^{\circ} 27201$ ).

Desde tal perspectiva, una gestión de la institucionalidad que se adecue a las pautas del derecho internacional de los derechos humanos debe procurar la integralidad de las acciones de los diferentes niveles de gobierno. Ello contribuye a evitar la superposición de acciones y el uso desproporcionado de los recursos (de diferente tipo) asignados en los presupuestos (Repetto, 2005; 2010). En tal sentido, la normativa propone una regulación adecuada sobre el modo de funcionamiento de la institucionalidad deportiva que comprende, puesto que involucra a los diferentes niveles de gobierno y establece las funciones correspondientes a cada uno de ellos.

De entre las medidas establecidas por los documentos oficiales que revisamos se agrega a la Asignación Universal por Hijo para Protección Social una asignación por hijo en 
el deporte. Dicho programa social es una transferencia monetaria condicionada que inició su recorrido en Argentina a partir del año 2009. A cada uno de sus destinarios menores de 18 años de edad (entre otros criterios) se les asigna una prestación dineraria en efectivo a cambio del cumplimiento de condicionalidades (a veces de modo personal y otras de parte de sus padres) educativas y de salud. A ello se agrega una asignación en el deporte para quienes estén interesados en tales actividades. En el respectivo documento se menciona: “... y en lo social incluyendo, mediante la Asignación Universal por Hijo en el Deporte, el derecho a la práctica del deporte y la actividad física de niños, niñas y adolescentes" (art. 1 de la Ley $N^{\circ}$ 27201). Si bien se trata de una regulación que en materia de juventudes reconoce el derecho a las prácticas deportivas, muchos de sus aspectos no han sido diseñados desde el enfoque de derechos humanos. Tal perspectiva es la que se propone desde el documento oficial. Sin embargo, es un programa focalizado y por consiguiente no universal, pues solo pueden acceder quienes reciben la Asignación Universal por Hijo, cuyos criterios de focalización ya explicitamos, y tengan entre 6 y 16 años de edad. Asimismo, y desde la asociación del enfoque de derechos y el de ciudadanía social, las condicionalidades colocan al destinario frente a la situación de cumplir con exigencias burocráticas que suponen un contrato desigualitario con el Estado (Fraser y Gordon, 1992) y eso sucede con esta asignación. En el documento se explicita del siguiente modo:

A los efectos del cumplimiento de las disposiciones del inciso e), del artículo 14 ter de la ley 24.714 y sus modificatorias, deberá acreditarse además la concurrencia de personas menores de edad y las personas discapacitadas obligatoriamente a asociaciones civiles deportivas incluidas en la ley 20.665 (art. 5 de la Ley $N^{\circ} 27201$ ).

Por otra parte, la normativa que regula esta nueva asignación para favorecer la práctica de actividades deportivas es incompatible con el cobro de otro tipo de becas y subsidios deportivos, ya sean del ámbito local o nacional. Tales incompatibilidades restringen aun más los criterios de focalización y no posibilitan el acceso a otras prestaciones dinerarias que pueden cubrir otro tipo de gastos vinculados con las prácticas deportivas, tales como indumentaria, viajes, cursos de formación.

En el marco de los documentos también se crea el Programa Nacional de Empoderamiento de la Mujer en el Deporte. Dicho programa comprende entre sus objetivos:

a) lograr el acceso equitativo de la mujer en el deporte; b) asegurar la formación con perspectiva de género de profesionales del deporte en los ámbitos federativos; c) promover la equidad en formatos competitivos, distribución geográfica, visibilidad y recompensas de los deportes de competición; d) concientizar y postular un trabajo de construcción colectiva en el ámbito de las federaciones deportivas, como posibles 
vehiculizadores de situaciones de violencia familiar o abuso sexual infantil en cuestión a la inmediatez y desarrollo del trabajo formativo que el deporte supone en niñas, niños y adolescentes (art. 8 de la Ley $N^{\circ} 27201$ ).

Desde una perspectiva de derechos humanos ligada a la reducción de brechas de género, todas estas instancias de socialización -en donde se construyen relaciones sociales de género- en las que se procure la incorporación de una mirada a partir de la cual se puedan considerar las particularidades de las condiciones en que viven las mujeres, sin dudas resulta primordial, pues es allí donde se produce esa distribución asimétrica de poder y de recursos. Ahora bien, si procuramos incorporar tal perspectiva, en referencia a los derechos humanos, debe considerarse tanto la igualdad formal como la igualdad material de las mujeres. Esta última es la que permite reparar en la adopción de medidas especiales de igualación entre géneros (Pautassi, 2007). De allí que si una mujer se interesa por realizar prácticas deportivas es probable que deba relegar sus tareas de cuidado en otros espacios o personas. Tales tareas han sido históricamente asignadas al género femenino y continúa siendo de ese modo. No solo ello, sino que frente a diferentes oportunidades (en este caso su incorporación en prácticas deportivas) el tiempo que demanda cuidar funciona como un obstáculo que obtura su posibilidad de elegir libremente. Ya desde hace varios años Amartya Sen (1999) afirmaba el vínculo entre desarrollo y libertad, pero especialmente entre ambos y la capacidad de agencia.

Un estudio realizado recientemente en Argentina analiza los resultados de las encuestas sobre el uso del tiempo en el país (en un periodo que comprende 2005 y 2010) y menciona que las mujeres ocupan su tiempo principalmente en tareas de trabajo doméstico no remunerado para su propio hogar, y dedican a esas actividades el doble de tiempo que los varones. Cuando tales mujeres logran insertarse en el mercado laboral -siempre en condiciones disímiles a los varones- continúan dedicándose a las tareas de cuidado resignando tiempos de ocio y descanso. Asimismo, cuando varones y mujeres que integran un mismo hogar participan en el mercado laboral, la distribución de tareas de cuidado es asimétrica en favor de los primeros y ello aumenta ante la presencia de niños en el hogar (Zibecchi, 2014).

Al mismo tiempo, podemos notar el desacierto de dedicar una sección especial -por paradójico que resulte- a la institucionalidad deportiva dirigida a las mujeres. Si bien se ha insistido mucho en la necesidad de adoptar acciones positivas (o de discriminación positiva) que logren igualar condiciones entre los diferentes géneros, en este caso no es necesario, por cuanto se coloca a las mujeres como un colectivo particular en lugar de considerar que en el tratamiento de los aspectos referidos a los jóvenes o a los adultos las relaciones sociales de género -como en toda relación social- se presentan estableciendo discriminaciones. De 
allí que sería apropiado que el documento incorporara una mirada transversal en donde fuera posible integrar la dimensión de la igualdad, y en ello la de género, en todas las acciones propuestas y desde la etapa de su diseño hasta la de su implementación y posterior evaluación (Astelarra, 2004).

Por otra parte, y tal como menciona la normativa del programa, se plantea entre sus objetivos el de concientizar sobre la posibilidad -dada la proximidad y los vínculos que se construyen en tales espacios de socialización- de advertir acerca de posibles situaciones de violencia o abuso hacia jóvenes o mujeres. Si bien ello deviene en una actividad relevante y de precaución, no deja de contribuir al riesgo individualizante de acciones que son propias del Estado y no de sujetos particulares. Esas actitudes estatales ya son habituales en materia de salud y medio ambiente por medio de las denominadas campañas de concientización ciudadana.

Si la competencia en el marco del deporte constituye la modalidad dominante en que se estructuran tales actividades, la presencia estatal (con toda la infraestructura institucional que ello supone) podría contribuir a construir una alternativa. Sin embargo, ello no sucede. Desde los orígenes de la consolidación de la nación argentina los criterios meritocráticos (bajo diferentes disfraces y algunos más elegantes que otros) fueron los que primaron, ya sea entre los integrantes de la Primera Junta del 25 de mayo de 1810 (que precedió a la declaración de la independencia) o entre quienes redactaron el Acuerdo de San Nicolás (que precedió a la Constitución Nacional) o entre quienes forjaron varios aspectos de los incorporados en dicha constitución. De este modo, criterios meritocráticos, establecidos explícitamente, son introducidos en el sistema de otorgamiento de subsidios y becas al deporte, regulado por medio de la Resolución № 1025 del año 2012 correspondiente al Ministerio de Desarrollo Social. En el documento se establece:

Se entiende por subsidios y subvenciones a los efectos del presente reglamento, a todas aquellas asignaciones y/o erogaciones extraordinarias o permanentes -con cargo de rendición de cuentas-, recibidas por las personas comprendidas en el artículo $4^{\circ}$ del presente reglamento, fundadas en razones de mérito u oportunidad, sin compensación, ni contraprestación económica o de otra especie, o que cuando éstas existan, fueren manifiestamente insuficientes o desproporcionadas al beneficio recibido (art. 2 de la Resolución № 1025/12 del Ministerio de Desarrollo Social).

Y continúa:

Se entiende por becas a los aportes en dinero de carácter no remunerativo que en virtud del artículo 1ำ, inciso g), del Decreto № 101/85 y sus modificatorios, se asignen por una o más veces o en forma periódica a deportistas aficionados que se destaquen 
en su especialidad, como apoyo para su entrenamiento y preparación. Tendrán prioridad los deportistas que integren equipos de representación nacional o que compitan individualmente en representación nacional (art. 3 de la Resolución $N^{\circ}$ 1025/12 del Ministerio de Desarrollo Social).

Si bien los criterios meritocráticos rodean a la mayoría de los sistemas de subsidios y becas a instituciones e interesados particulares, en muchos casos se han incorporado otro tipo de criterios -con el propósito de atenuar el efecto de los primeros- tales como la situación socioeconómica del postulante, la vacancia de ese tipo de becas en el lugar de origen de la institución o el postulante, las condiciones socioambientales del territorio en donde se solicita el subsidio o habita el postulante. En tal sentido, y con el propósito de aminorar los dominantes criterios meritocráticos, en el marco de la institucionalidad deportiva prevista por la Ley N²7201 se crea el Programa de Becas Sociales Deportivas. Dicho programa tiene como objetivo la capacitación y preparación de deportistas y técnicos que integren equipos representativos nacionales de disciplinas deportivas noveles o federadas que compitan en competencias federadas o sociales de carácter nacional. A priori no parece regirse por tales criterios aunque la mayoría de los aspectos que propone quedan sujetos a una futura regulación de la ley por medio del dictado de otro documento oficial.

Vale mencionar que en la relación entre derechos y meritocracia, el aspecto de igualdad material o sustancial de los primeros es en donde debe priorizarse -tal como señala el sociólogo francés François Dubet (2011)- una igualdad de posiciones y no de oportunidades, en donde puedan aproximarse las diferencias estructurales (económicas 0 culturales) que caracterizan las posibilidades de desarrollo y de acceso a los recursos.

\section{Conclusión}

Dado que tanto derechos humanos como inclusión social se han convertido en significantes recurrentes por parte del discurso político y mediático, ello amerita un análisis libre de banalidades a cargo de las ciencias sociales. Sin duda, ambos conceptos -tal como ya lo hemos explicitado- se encuentran vinculados, puesto que los derechos proponen la inclusión por medio de políticas sociales universales, y por consiguiente no focalizadas, dirigidas a todos los sectores de la población, aunque con distinciones respecto de aquellos sectores en los que persistan situaciones de discriminación, tal como sucede con las juventudes o las mujeres.

Del análisis de la institucionalidad deportiva para jóvenes y mujeres podemos advertir que pendula en torno a dos conceptos: derechos y meritocracia. El primero de ellos se objetiva en importantes medidas sociales y el modo en que se ha previsto su ejecución, por su parte, el segundo, en un particular mecanismo de acceso a determinados subsidios y 
becas. Una de tales medidas es la creación de una asignación universal por hijo en el deporte que se implementa en el marco de un programa social más amplio y con mayor trayectoria que este. Si bien se incorpora una nueva prestación en el marco de una política social, no deja de ser un programa focalizado y que solo cubre necesidades básicas para el ejercicio de la práctica deportiva. También se crea el Programa Nacional de Empoderamiento de la Mujer en el Deporte, el cual constituye una importante medida en términos de derechos humanos y género pero que, como ya hemos explicitado, no tiene en cuenta las condiciones materiales en que viven las mujeres, de allí que muchas de las oportunidades que propone serán obturadas por las mismas.

A pesar de estos importantes avances en materia de derechos y de inclusión social, aún existen aspectos meritocráticos en donde una vez más la competencia desigual por el acceso a determinados recursos se hace presente. Ello puede advertirse particularmente en el sistema de subsidios y becas al deporte previsto en el marco de dicha institucionalidad. Si bien existen intentos por aminorar los efectos meritocráticos mediante la creación de un programa de becas sociales, todavía no se encuentra reglamentado y de allí que no puede conocerse en detalle las medidas que propone.

\section{Referencias bibliográficas}

Andrenacci, L. (2010). Política social y ciudadanía en América Latina contemporánea: una contribución al debate acerca de las transformaciones de política social de la región en la primera década del Siglo XXI. Ponencia presentada en el II Congreso Latinoamericano y Caribeño de Ciencias Sociales. México.

Astelarra, J. (2004). Políticas de género en la Unión Europea y algunos apuntes sobre América Latina. Santiago de Chile: CEPAL-Naciones Unidas.

Becher, Y. (2018). Una propuesta de estudio de procesos de inclusión y acceso a derechos en juventudes y género. Revista de Direito da Cidade. Universidade do Estado do Rio de Janeiro. En prensa.

Braun, V. y Clarke, V. (2006). Using thematic analysis in psychology. Qualitative Research in Psychology, 3(2), 77-101. Bristol: University of the West of England.

Burchardt, T., Le Grand, T. y Piachaud, D. (2002). Degrees of exclusion: developing a dynamic, multidimensional measure. En J. Hills, J. Le Grand and D. Piachaud (Eds.). Understanding social exclusion. (pp. 30-43). Oxford: Oxford University Press.

Castel, R. (2004). Encuadre de la exclusión. En S. Karsz (Coord.). La exclusión: bordeando sus fronteras. Definiciones y matices. (pp. 55-86). Barcelona: Editorial Gedisa. 
Delor, F. y Hubert, M. (2000). Revisiting the concept of vulnerability. Social science and medicine, 50, 1557-1570.

Dubet, F. (2011). Repensar la justicia social. Contra el mito de la igualdad de oportunidades. Buenos Aires: Siglo XXI Editores.

Duverger, M. (1981). Métodos de las ciencias sociales. España: Ariel. Traducción de Alfonso Sureda.

Fraser, N. y Gordon, L. (1992). Contrato versus caridad: una reconsideración de la relación entre ciudadanía civil y ciudadanía social. ISEGORÍA, (6) 65-82. Traducción de Pedro Francés Gómez.

Levitas, R. (2006). The concept and measurement of social exclusion. En Pantazis, C., Gordon, D. and Levitas, R. Poverty and social exclusion Britain. (pp. 123-160). Bristol: The policy press.

Maxwell, J. (1996). Qualitative research design. An interactive approach. New York: Sage Publications.

Oszlak O. y O’Donnell, G. (1995). Estado y políticas estatales en América Latina. Redes. Revista de Estudios Sociales de la Ciencia, 4(2), 9-128. Universidad Nacional de Quilmes.

Pautassi, L. (2007) ¡Cuánto trabajo mujer! El género y las relaciones laborales. Buenos Aires: Capital Intelectual.

Repetto, F. (2005). La dimensión política de la coordinación de programas y políticas sociales: una aproximación teórica y algunas referencias prácticas en América Latina. En F. Repetto (Ed.). La gerencia social ante los nuevos retos del desarrollo social en América Latina. Guatemala: INDES-Guatemala.

Repetto, F. (2010). Protección social en América Latina: la búsqueda de una integralidad con enfoque de derechos. Revista del CLAD Reforma y Democracia, 47, Venezuela.

Sen, A. (1999). Development as Freedom. Oxford: Oxford University Press.

Tonon, G. (2015). Relevance of the use of qualitative methods in the study of quality of life. En G. Tonon (Ed.). Qualitative Studies in Quality of Life. Methodology and practice. (pp. 3-21). New York: Springer.

Towsend, P. (1979). Poverty in the United Kingdom. Harmondsworth: Penguin.

Vázquez, M. (2015). Juventudes, políticas públicas y participación: un estudio de las producciones socioestatales de juventud en la Argentina reciente. Buenos Aires: Grupo Editor Universitario. 
Zibecchi, C. (2014) ¿Cómo se cuida en Argentina? Definiciones y experiencias sobre el cuidado de niños y niñas. Buenos Aires: Equipo Latinoamericano de Justicia y Género.

\section{Otras fuentes consultadas:}

Consejo Latinoamericano de Ciencias Sociales (CLACSO). Organización de las Naciones Unidas para la Educación, la Ciencia y la Cultura (UNESCO). (2013). Políticas de inclusión social de jóvenes en Latinoamérica y el Caribe: situación, desafíos y recomendaciones para la acción. Consultada el 15 de Noviembre de 2015, http://juventude.gov.br/articles/participatorio/0005/7083/INFORME CLACSO UNES CO.pdf

Comité para la Eliminación de la Discriminación contra la Mujer (2010). Observaciones finales para Argentina. Organización de Naciones Unidas.

Comisión Económica para América Latina y el Caribe (CEPAL). Observatorio de Igualdad de Género de América Latina y el Caribe (2017). Planes de igualdad de género en América Latina y el Caribe. Mapas de ruta para el desarrollo. Santiago de Chile: Naciones Unidas.

Foro Económico Mundial (2016). Índice Global de la Brecha de Género 2016. Consultada el 04 de Mayo de 2017, http://www.datosmacro.com/demografia/indice-brecha-generoglobal

Instituto Nacional de Estadísticas y Censos (INDEC) (2015). Encuesta Nacional de Jóvenes 2014. Principales resultados. Argentina.

Organización Iberoamericana de la Juventud (OIJ). Comisión Económica para América Latina y el Caribe (CEPAL-ONU) (2015). Juventud Iberoamericana 2015. Hoja mural de datos sociodemográficos.

\section{Fuentes normativas:}

República Argentina. Honorable Congreso de la Nación Argentina. Asignaciones Familiares Ley $\mathrm{N}^{\circ}$ 24714. Sancionada: 2/10/1996. Promulgada Parcialmente: 16/10/1996. Publicada en el Boletín Oficial n² 28503 del 18/10/1996, p. 1.

República Argentina. Honorable Congreso de la Nación Argentina. Programa Social y Deportivo “Juegos Nacionales Evita". Ley No 26462. Sancionada: 10/12/2008. Promulgada de Hecho: 7/01/2009. Publicada en el Boletín Oficial $n^{\circ} 31572$ del 14/01/2009, p. 1 
República Argentina. Honorable Congreso de la Nación Argentina. Ente Nacional de Desarrollo Deportivo. Ley $\mathrm{N}^{\circ}$ 27201. Sancionada: 28/10/2015. Promulgada: 03/11/2015. Publicada en el Boletín Oficial n³3249 del 04/11/2015, p. 11.

República Argentina. Poder Ejecutivo Nacional. Subsistema no contributivo de "Asignación Universal por Hijo para Protección Social". Decreto № 1602/09. Decretado el 29/10/2009. Publicado en el Boletín Oficial n³1770 del 30/10/2009, p. 1.

República Argentina. Poder Ejecutivo Nacional. Ministerio de Desarrollo Social. Reglamento de Subsidios, Subvenciones y Becas destinadas al Fomento del Deporte. Resolución $N^{\circ}$ 1025/12. Resuelto el 13/03/2012. Publicada en el Boletín Oficial $n^{\circ} 32381$ del 19/04/2012, p. 9. 